\title{
Teleoperated Visual Inspection and Surveillance with Unmanned Ground and Aerial Vehicles
}

\author{
doi:10.3991/ijoe.v4i4.699 \\ Hartmut Surmann ${ }^{1}$, Dirk Holz ${ }^{2}$, Sebastian Blumenthal ${ }^{2}$, \\ Thorsten Linder ${ }^{2}$, Peter Molitor ${ }^{1}$ and Viatcheslav Tretyakov ${ }^{2}$ \\ ${ }^{1}$ Fraunhofer Institute for Intelligent Analysis and Information Systems (IAIS), St. Augustin, Germany \\ ${ }^{2}$ University of Applied Sciences Bonn-Rhein-Sieg, St. Augustin, Germany
}

\begin{abstract}
This paper introduces our robotic system named UGAV (Unmanned Ground-Air Vehicle) consisting of two semi-autonomous robot platforms, an Unmanned Ground Vehicle (UGV) and an Unmanned Aerial Vehicles (UAV). The paper focuses on three topics of the inspection with the combined UGV and UAV: (A) teleoperated control by means of cell or smart phones with a new concept of automatic configuration of the smart phone based on a RKIXML description of the vehicles control capabilities, (B) the camera and vision system with the focus to real time feature extraction e.g. for the tracking of the UAV and (C) the architecture and hardware of the UAV.
\end{abstract}

Index Terms-USAR, teleoperation, UGV, UAV, OCU, visual attention, computer vision

\section{INTRODUCTION}

Mobile robotic systems for tele-exploration are gaining more and more importance, especially for industrial inspection tasks and rescue operations. In scenarios, like those that are addressed e.g. in Urban Search And Rescue (USAR), fully autonomous systems are not applicable because of safety or efficiency reasons. Here, human operators control semi-autonomous robot systems to gain information about environments. Robotic systems act as proxies for humans in environments where humans can not or are not allowed to enter. Basically, research efforts have been focused on the following areas:

- Mobility and robustness of the robot platform,

- Development of reliable and accurate sensors for mobile robots and

- Human-Machine-Interfaces with high usability and acceptance for the human operator.

As every platform has its own advantages and disadvantages, they also have different applications and workspaces where they are particularly suitable. Hence, it is reasonable to use several platforms concurrently for the same task combining their individual strengths, i.e. so called multirobots [1]. These cooperative systems are composed of several heterogeneous robots, e.g. a smaller mobile robot that is carried by a larger one and can be dropped off in cases where the larger robot can not further explore the environment because of its size. The larger robot, on the other hand, can carry different tools and batteries for the small-size robot. Recently, research groups have started to address the combination of ground and aerial vehicles. Whereas ground vehicles can enter e.g. collapsed building or mines, aerial vehicles can help to get an overview of the whole site [2].

One major problem is to deliver the required information about the surrounding of the robot to the operator. Cameras, mounted on Unmanned Ground Vehicles (UGVs) or Unmanned Aerial Vehicles (UAVs), have become the de facto standard sensors to provide this information. Our intention is to develop a platform to increase the level of reconnaissance during a USAR operation. To achieve this goal it is reasonable to combine a ground and an aerial vehicle. Here we introduce our approach named UGAV (Unmanned Ground-Air Vehicle) of a robotic system consisting of two semi-autonomous robot platforms, an UGV (see Fig. 1) and an UAV. Both robots are equipped with camera systems for surveillance. The operator can directly control the UGV and the UAV (see Fig. 6). Furthermore the aerial vehicle can be commanded by the UGV for autonomous missions e.g. sending GPS coordinates which have to be observed and for autonomous landing.

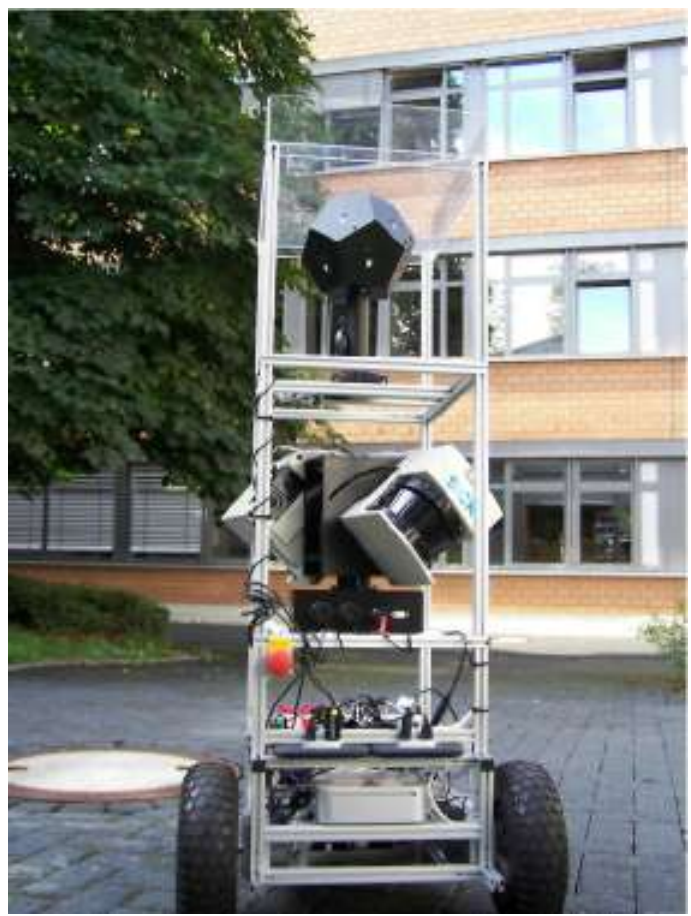

Figure 1. First evaluation of the UGAV. The ground platform is built of a Volksbot RT3 which is equipped with batteries, vision sensors, 3D laser scanner, control computers and a landing platform on top for the quadrotor 

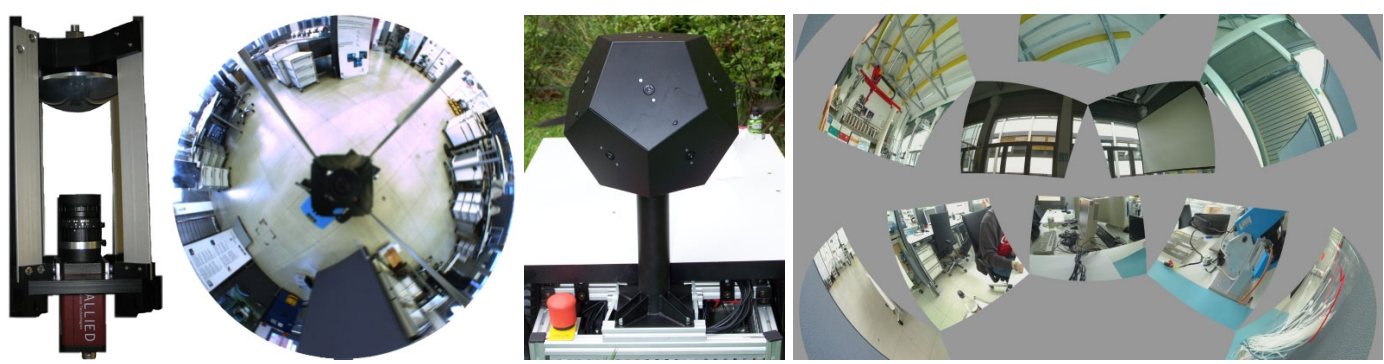

Figure 2. Visions systems for the ugv. (a) shows the omnidirectional IAIS vision system. The camera aims towards a hyperbolic mirror. (b) is an image from the perspective of the omnidirectional camera. (c) The dodecahedron shaped camera system with eleven cameras. each camera aims in another direction. (d) demonstrates an impression from panoramic images of the dodecahedron.

Especially for autonomous landing the ground vehicle has to detect and track the aerial vehicle. We present a real-time visual attention approach to track the UAV. The camera views from the vehicle are also presented to the operator. For teleoperating the UGV we use an off-theshelf mobile device.

The paper is organized as follows: Chapter 2 gives a brief overview on the used UGAV hardware, whereas the teleoperation for the overall system is described in Chapter 3. Chapter 4 copes with acquisition of visual data and extraction of spatial information out of our new camera concept of a dodecahedron cube or a higher resolution omnidirectional camera. The architecture and control issues of the aerial vehicle are described in chapter 5. Chapter three, four and five give a brief state of the art at the beginning of those chapters.

\section{Platform}

Our robotic system (see Fig. 1) consists of an UGV and UAV which are briefly described in the following sections.

\section{A. Unmanned Ground Vehicle}

The UGV is based on a modular mobile platform called VolksBot [3], which has been designed specifically for rapid prototyping and applications in education, research and industry. The VolksBot system is developed, manufactured and sold by the Fraunhofer Institute for Intelligent Analysis and Information Systems (IAIS). It easily allows access to and replacement of several components such as motors, batteries and electronics as well as the extension of new hardware ${ }^{1}$. For stability reasons in rough terrain we have chosen the six-wheeled version VolksBot RT6 (see Fig. 3) out of the several variants of the VolksBot [4]. It has a size of $700 \mathrm{x} 480 \mathrm{x}$ $600 \mathrm{~mm}(\mathrm{LxWxH})$ and a weight of approx. $15 \mathrm{~kg}$. As all six wheels are driven by the two $150 \mathrm{~W}$ motors, the robot is even able to climb smaller stairs or steps. The robot has a maximum velocity of $1.1 \mathrm{~m} / \mathrm{s}$ and a maximum payload of approx. $40 \mathrm{~kg}$. For indoor applications front and rear wheels can be chosen to have no tread pattern to enhance rotation. Two MAC Minis (CPU 2GHz, Memory 2GB) serve as computational units for processing sensor data and controling the UAV.

1 http://de.youtube.com/watch?v= 7ACC8-KYQY, http://de.youtube.com/watch?v=lI_f7v4meBU
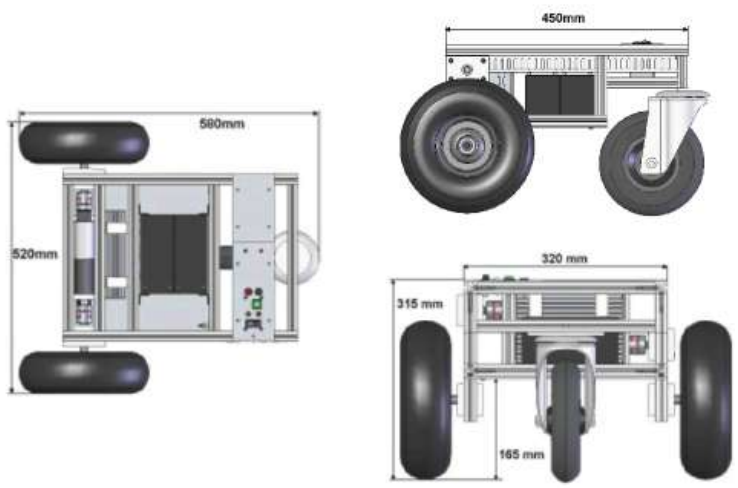

Figure 3. Engineering drawing of the Volksbot RT3 chassis. RT3 is a three-wheeled robot platform. The left sketch shows top view, upper right shows lateral view and lower right shows front view.

\section{B. Unmanned Aerial Vehicle}

The UAV is a four-rotors aerial platform, a so-called quadro-tor [6], that is capable of Vertical Take-Off and Landing (VTOL). Its flight control board is equipped with an inertial measurement unit consisting of 3-axes gyroscopes, 3-axes inertial sensors, 3-axes digital compass and a GPS module. For altitude control a pressure sensor is employed. Fusion of these sensors as well as the control of the four motors is done by means of an on-board 20 MHz-microcontroller (Atmel ATMEGA644P) and four brushless motor control boards. The on-board microcontroller communicates with the four brushless controllers via $\mathrm{I}^{2} \mathrm{C}$ bus.

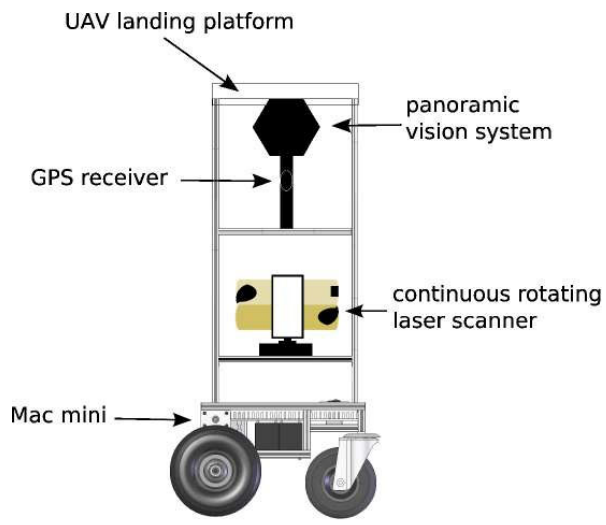

Figure 4. Schematic overview of the evaluation platform volksbot. It is equipped with a panoramic vision system (sphere cube with 11 cameras), a continuous rotating 3d laser scanner, a GPS receiver, a landing platform for the quadrotor, batteries and control computers. 
The quadrotor has a size of $650 \times 650 \times 220 \mathrm{~mm}(\mathrm{~L} \times \mathrm{X}$ $\mathrm{x} \mathrm{H}$ ) and a weight of $590 \mathrm{~g}$. With an extra antenna the height increases to $550 \mathrm{~mm}$ and the weight increases to 620 g. With fully loaded batteries (2100 mAh) it can operate approx. $20 \mathrm{~min}$. Its maximum payload is $350 \mathrm{~g}$. The quadrotor is controlled either by the UGV or a human operator via $\mathrm{WiFi}$, Bluetooth or an analog remote control unit. The architecture of the quadrotor is further explained in section $\mathrm{V}$.

\section{Vision sensors}

For the USAR purpose the RT3 is equipped with one of the following vision systems. The first one consists of the IEEE 1394 firewire camera "AVT Marlin F-145-C" aiming towards a hyperbolic mirror. This camera can deliver up to 10 frames per second in high resolution color mode (1392 x 1038 pixels). The second vision system is built up from eleven off-the-shelf USB-webcams aiming in different directions. They are mounted in a dodecahedron shaped chassis with a size of 220 x 220 x $380 \mathrm{~mm}(\mathrm{~L} \times \mathrm{W} \times \mathrm{H})$. Each camera delivers up to 15 frames per second at a resolution of $800 \times 600$ pixels. At a lower framerate, pictures with a resolution of $1600 \times 1200$ pixels can be acquired. All eleven cameras are connected to the Mac minis via USB 2.0. Both platforms are suitable for teleoperated applications like USAR or visual surveillance scenarios.

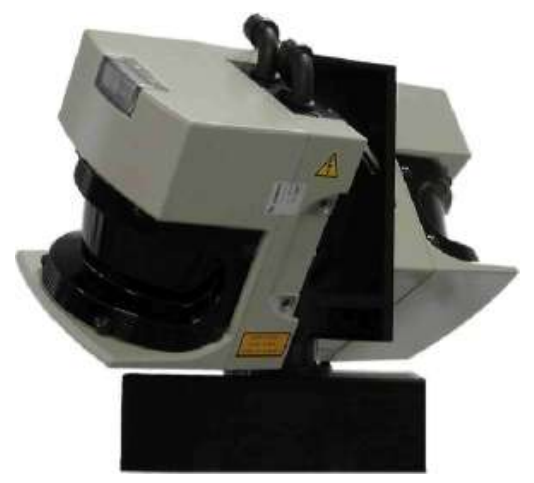

Figure 5. The continuously rotating laser scanner 3DLS-K2 consists of a rotation unit with 2 SICK-LMS laser scanners.

\section{3D Laser scanner}

For building precise geometric maps the UGAV is equipped with a continuously rotating 3D scanner (3DLSK2 Fig. 5, [5]). The 3DLS-K2 consists of a rotation unit to reach the 3rd dimension and of two 2D time-of-flight SICK laser scanner (scanning range $180^{\circ}$ ). They can provide distance measurement in a range of up to $80 \mathrm{~m}$ with a centimeter accuracy. The continuously yawing scan scheme [7] of the unit generates two 360 degree dense point clouds every 1.2 seconds. The hundreds of $3 \mathrm{D}$ point clouds acquired during a robot run are registered in a common coordinate system with the well known ICP (iterative closest point) algorithm described in several previously published papers e.g. $[8,9]^{2}$.

\section{TELEOPERATED ROBOT CONTROL}

A teleoperator is a physical device which is enabling an operator to move around, sense or mechanically manipulate objects by using a robot. These devices can be

\footnotetext{
${ }^{2}$ See http://de.youtube.com/watch?v=xr28pX9ZkXw
}

separated into two device classes. Both have in common that the teleoperators allow spatially separating the operator from the machine and the robot $[10,11]$.

The first class is named "anthropomorphic" which means that these teleoperators have a manlike physiognomy. Anthropomorphic teleoperators are mostly used in combination with a manipulator to allow a remote handling of objects (e.g. handling toxic or radioactive waste). The "non-anthropomorphic" devices build the second class. This class includes a lot of different device types like PCs, laptops, special handheld controllers and vehicle cockpits [12]. If the teleoperator is a laptop or a similar device they are also called Operator Control Units (OCU).

OCUs are common for USAR robots and often built into waterproof boxes [11]. This mounting concept respects two essential requirements for the whole rescue equipment. The first one is given by the fact that every object which is used on a mission must be either able to be easily decontaminated or must be disposed to ensure that no biologic, toxic, chemic or radioactive contamination can effect the rescue teams or the population [13]. The second is given by the need of portability and robustness. The rescue team member must be able to carry the OCU and it can not be ensured that the OCU will not be dropped during a march to the operational area.

Each teleoperator needs a connection to its robot and widely varied techniques are in use to ensure optimal communication channels with or without respecting real time requirements [14]. There are different technologies available, either wired or wireless connections. As shown in $[11,13]$ the usability of wireless techniques is limited in the situation of exploring small voids from a collapsed building. Based on the nature of radio frequency transmission, the signal can be heavily disturbed or poor in such an environment. This might cause that the robot gets lost, as seen on the World Trade Center catastrophe [11].

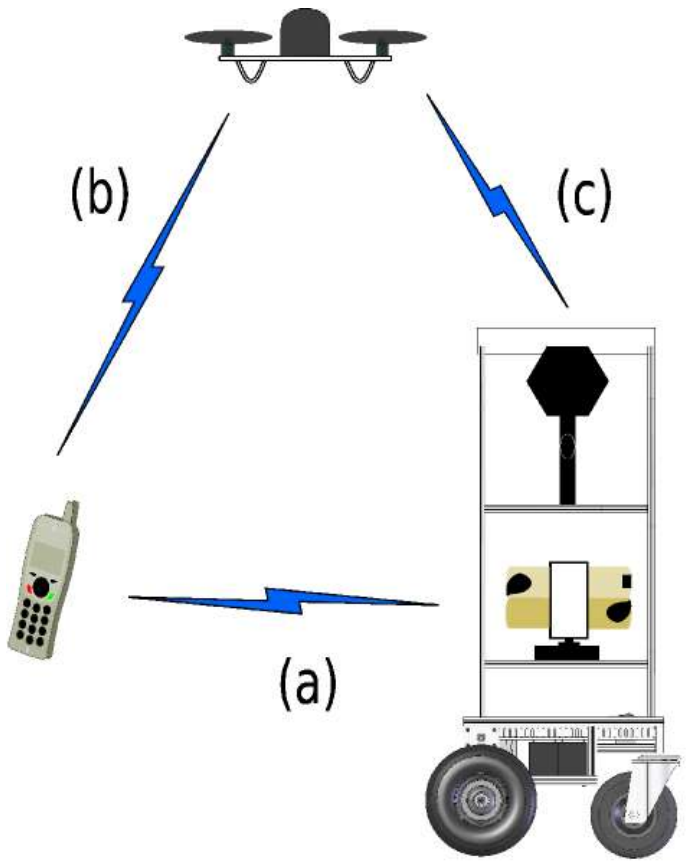

Figure 6. Unmanned ground-air vehicle (UGAV): the mobile device controls either the UGV (a) or the UAV (b). (c) UAV is commanded by the UGV. 
The advantage of a wireless communication technique is the complete physical isolation between the robot and the operator. Data- and safety-tether might get caught and reduce the freedom of movement. Hence, the decision on which technique should be used heavily depends on situation and task.

\section{A. Mobile device (PDA or Cellphone)}

Human robotic interaction is still a wide open space for research and is gaining more and more importance. Mobile devices like PDA's or cell phones, which accompany us anywhere and at anytime, are the most convenient tools to help us beneficing from ubiquitous service provided by mobile robots. The latest mobile devices are offering more multimedia features, better communication capabilities (Bluetooth, Wi-Fi, GPRS/UMTS) and are easier programmable than before $[15,16]$. So far, those devices have been used mainly for communication, entertainment, and as electronic assistants. In other words, mobile devices are at the moment one of the best candidates to intermediate between us and our surroundings.

Therefore, instead of using an ordinary Laptop or a special remote device to control the robot, our approach is to use mobile devices like PDAs or smartphones. This concept is just starting and not quite common at the moment. However, it has multiple advantages out of the shelf. In fact there is a widespread research field evaluating the beneficing and usability of mobile devices (e.g. [17-19]):

- Always available: Cell phones are nowadays widespread and there are already trials to make them part of the normal rescue worker equipment [20]. Therefore the devices are already up on the ground and can also be used for controlling the robot.

- High social acceptance and limited teaching: Handling of the OCU is one of the strongest barriers which detain rescuers to make use of the advantages or their robots. They have to be trained to use and interpret sensors of the system [11]. This training can be limited if they already know how the physical device works. This is given for mobile phones.

- Man-packable, light weight and size: In an urban catastrophe scenario the equipment have to be man portable. This means that rescue workers have to be able to transport their technical equipment to the ground of interest by themselves. Both, the OCU and the robot have to be carried. An OCU which is based on a mobile device like a cell phone does not have these requirements (they are still on the ground) and can be easily carried by the same person supplying the robot.

- Long run time: A long runtime is required for USAR for both the OCU and the robot as mentioned in [13]. Cell phones are able to operate for a sufficient time.

- Robustness and substitutability: Mobile devices are robust enough for the daily use. But they are not designed to be for rough handling or to be waterproof. These disadvantages can be managed by using special cases for the mobile devices to fit the requirements [21]. Cell phones are out of the shelf products, therefore they are not as expensive as common OCUs. Following this fact mobile devices are more substitutable than OCUs.

- General purpose utilizable concept: Millions of mobile robots are actually driven in-house starting from small LEGO toys up to vacuum cleaners or service robots and their number tends to increase. Therefore the need of interaction between such systems and humans is growing. Cell phones are available to do these jobs and it is expected that they will strongly influence our life [22].

Disadvantages of using mobile devices for teleoperation are the limited computing power and small screen sizes. This reduces the usability of mobile devices to simple teleoperation tasks which is not always desirable. Furthermore, mobile devices are limited by the numbers of supported interfaces and are hardly extendable. Some of these disadvantages can be compensated by the capabilities of the robot. For example the limited computing power can be compensated partly by acquiring pre-computed data from the robots.

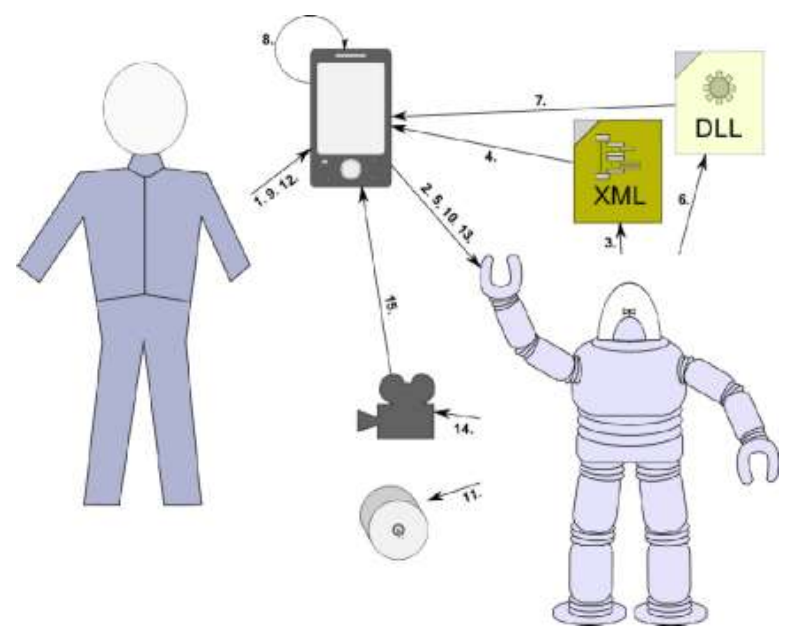

Figure 7. OCU and robot interaction overview. 1: An operator selects the robot on the mobile device, 2: OCU-software connects to robot and requests the RKI-file, 3-4: Robot sends description, 5: OCU-software requests control plugins, 6-8: Robot sends plugins, and OCU adapts its self, 9-10: Operator selects an action for the robot (e.g. move) and the OCU transmits the needed commands, 11: Robot receives and executes command, 12-13: Operator demands sensor data, 1415: Robot sends the sensor data...

\section{B. Android}

As a representative for the upcoming smart phone generation a simulation software development kit called Android is used. Android is a new platform for mobile devices like cell phones. The Open Handheld Alliance is developing Android as an open software Operating System (OS). The kernel of Android is a Linux-Kernel in version 2.6. The developers have setup a framework on top of the Kernel to guarantee that the whole OS can be used via JAVA applications. Therefore the main programming language (currently the only application language which can be used) is JAVA [23]. Other cell phone operating systems which are programmable in JAVA use mostly the JAVA mobile version J2ME. However, J2ME is functionally reduced and not as powerful as the normal JAVA (J2SE) [24]. The new framework makes use of the "Dalvik" Virtual Machine. This allows for using nearly the full standard JAVA 
libraries on mobile devices. A special advantage of Android is the open source concept which will allow extending the OS for the needs of USAR.

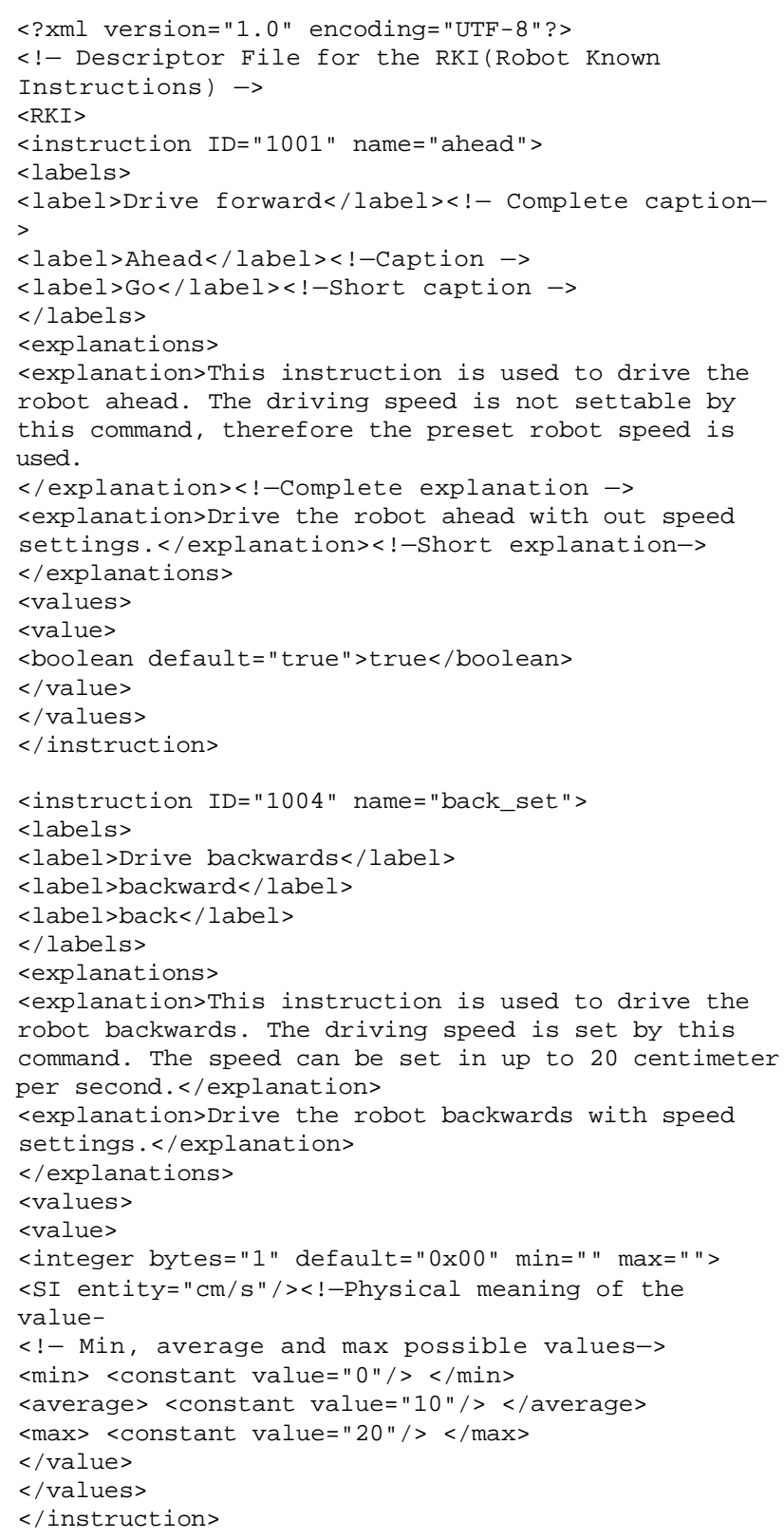

Figure 8. Example of a RKI (robot known instruction) file. The file is stored at the robot and is request by the control client.

The Android project includes support for GSM and UMTS telephone networks. Furthermore, it supports WiFi, Bluetooth and USB. Therefore the platform will allow several communication options. This fact allows us to use a widespread communication background (e.g. if a near location network like WiFi is not available the system can use long-distance networks like the GSM network).

The expected CPU-power, size, weight and runtime can be approximated by the current available smart phones. The computing power of smart phones is up to about 600 $\mathrm{MHz}$. This is far less than the current power of PCs or Laptops. These facts challenge our project and it is to be evaluated if the CPU-power can reach our goals. Nevertheless, first results indicate a positive outcome on that question. An additional positive effect by using cell phones in USAR is given by the growing popularity of the Global Position System (GPS) for these mobile devices. So, the most Android phones will have a built in GPS antenna which allows for determining the position of the Operator [25]. This information can be used to interact with the robot and provides basic means for homing and extended path planning routines.

\section{XML Robot Instructions}

Universal remote control information is not published by current mobile robots because they are controlled by special remote control devices. Cell phones on the other hand have different inhomogeneous operating systems and are widely used. To use them for guiding a mobile robot, the robot control parameters are stored in a XML file at the vehicle and distributed to control clients. Each cell phone can request a RKI (Robot Known Instruction) file to configure the control program and serve as a control client. Figure 8 shows an example of an RKI file. This method allows the use of the OCU-software on a wide number of robots (cf. [19,26]). The fundamental idea is that the robot informs the Operator and its OCU about its control information. Furthermore, to handle and interpret the transmitted robot data correctly the control program is extendable with plugins which are also transmitted by the robot server. Therefore, the OCU (cell phone) can upate to several different robots and handle different sensor data streams (Fig. 7). After the configuration with proper control plugins, the operator and robot are ready to interact. The outgoing operator commands are interpreted by the robot and the incoming sensor data is handled and presented to the operator at the OCU.

\section{First Results}

We use the Android simulator (version m5-rc15) to evaluate the usability of the platform for an OCU. The simulator is able to mimic the behavior of a mobile phone running Android [27]. The OCU software was written in JAVA and matches following use-cases:

- Open up a bidirectional wireless connection between a ground based robot and the OCU.

- Display the robot camera stream (unidirectional).

- Navigate the robot via basic command sets and a graphical user interface.

The outcome of the first test rides is publicly available as videos on [28] and [4]. As you can see there the Android simulator was running on a conventional notebook (Intel Mobile 1.73 GHz, 1 GB RAM) and used the IEEE $802.11 \mathrm{~b} / \mathrm{g}(\mathrm{WiFi})$ standard to setup a communication channel ${ }^{3}$. A three-wheeled robot (VolksBot RT3) was used during the testing period with same VolksBot Motor Controller (VMC) as on the RT3. The IAIS-Vision system on top allows a panoramic view (see Fig. 2(a)). The user interface is setup by respecting that also non-specialists must be able to control the robot. The whole interface can be controlled through buttons and the cell phones touch pad, if available. This makes the handling intuitive and respects the request to limit the need of training (see Fig. 9).

\footnotetext{
${ }^{3}$ http://de.youtube.com/watch?v=LKmCPMNUYZs
} 


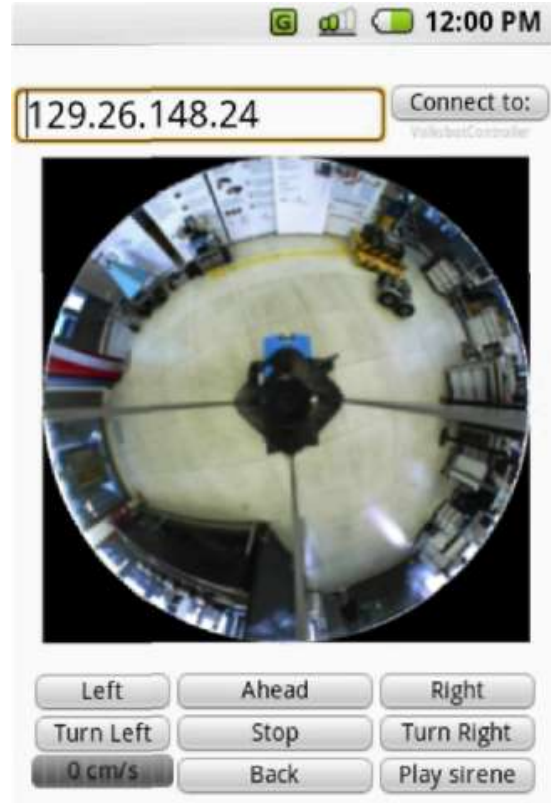

Figure 9. User interface of teleoperator software in the android environment.

The prototype OCU software is a single screen which is separated into three parts. On top of the screen the operator inserts the address of the robot and can start the connection via a button. In this prototype version of the OCU the address is based on IP4 and a local network domain name. The communication is similar to that of standard client-server architectures (robot: server; OCU: client).

Visual information is a fundamental for USAR. It is needed e.g. for navigation and finding victims [11,29-31]. As a result the camera view is displayed in the middle of the cell phone screen. This explicit region shows the image of the on board robot camera. If the robot is equipped with an omnidirectional camera system like the IAIS-Vision system the operator obtains a panoramic view over the surroundings. The bottom of the user interface is reserved for motion control. The OCU software allows for commanding translational and rotational velocities as well as some predefined motions like for instance turning on the spot (if available).

For safety reasons the robot is setup with a watchdog functionality. This means it decelerates if there is no new command within the last two seconds and stops immediately if the signal to the OCU gets lost. In our testing environment this safety functionality works well, but as seen in [11] this behavior is critical. An outcome of this safety behavior can be that the robot gets lost during a mission and may not be recoverable. Therefore this behavior is to be extended by the functionality of searching for an alternative communication channel and by autonomous homing skills.

\section{VISION SYSTEMS}

A fundamental problem in the field of vision for mobile robots is the online perception of the environment. The vision systems deliver an overview of the scene which supports the operators impression of the whole scenery and provides visual information for controlling and steering the semi-autonomous robot. Another crucial task comprises finding victims and inspection of buildings or cluttered terrain for revealing structural damages or hazardous areas [30]. Therefore the UGV is equipped with one of the following panoramic camera systems: (A) One camera aiming towards a hyperbolic mirror which enables a hemispherical but distorted view (see Fig. 2(a)) and (B) a dodecahedron cube consisting of eleven cameras aiming in different directions. They are mounted in a pentadodecahedron shaped polyhedron to achieve a near full spherical view (see Fig. 2(c)). The grabbed frames from the cameras are undistorted but the image processing results in higher computational efforts.

To improve the operator's scenery awareness we tested different feature extraction mechanisms, according especially to their real-time applicability. Image features can be used i.e. to calculated depth information (for mapping) if they occur in several different images or to help loop closing (for SLAM). Our approach is based on features in the image data. Figure 10 shows a schematic overview of the system.

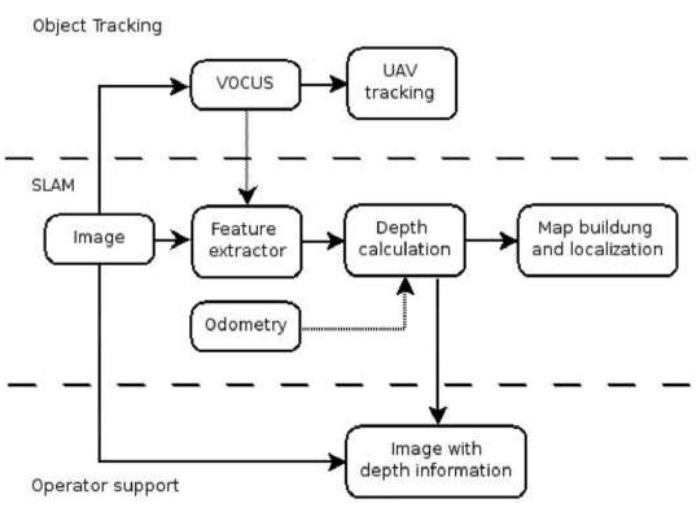

Figure 10. Schematic overview of the vision systems.

Features need to be robust against changes in scale, rotation and occlusion. Two widely used algorithms for the feature extraction are the SIFT algorithm from [32] and SURF algorithm from [33]. Both algorithms are robust against changes in scale, rotation and occlusion.

TABLE I.

COMPARISON BETWEEN SIFT- AND SURF-BASED FEATURE DETECTION ALGORITHMS. THIS IS DONE ON FULL RESOLUTION IMAGE AND ON A ROI IN THE IMAGE.

\begin{tabular}{|c|c|c|}
\hline Algorithm & Time per picture & Number of features \\
\hline \hline SIFT & $4300 \mathrm{~ms}$ & 2777 \\
\hline SURF & $1180 \mathrm{~ms}$ & 1475 \\
\hline SIFT (ROI) & $3100 \mathrm{~ms}$ & 147 \\
\hline SURF (ROI) & $391 \mathrm{~ms}$ & 116 \\
\hline
\end{tabular}

Sim et. al. [34] and also Karlson et. al. [35] demonstrates vision-based robot navigation systems using SIFT features, but only offline. The SURF algorithm provides comparable good results and needs less computational power because of utilizing integral images and approximated digital filters (Haar wavelet) but is also not real-time capable [29]. To recognize a feature in different images a unique descriptor is necessary. While the SIFT descriptor consists of a 128 dimensional vector SURF needs only 64 dimensions by default. Hence the shorter descriptor yields advantages in the nearest neighbor matching algorithm. The matched feature pairs, and the changes in their bearing due to the robots movement are consulted for calculating depth information 
$(\lambda)$. This is done by a triangulation-like linear algebra approach where $\mathcal{X}$ represents the current position in global coordinates and $\vec{a}$ and $\hat{b}$ are unit vectors describing the directions to the feature. $x_{1}(\lambda)=x_{01}+\lambda \cdot a$, $f_{2}(\kappa)=f_{02}+\kappa \cdot \stackrel{\mu}{b}$ and $\tilde{c}=\tilde{x}_{02}-\tilde{x}_{01}$ leads to the linear system of equations:

$$
\left(\begin{array}{cc}
a_{x} & -b_{x} \mid c_{x} \\
a_{y} & -b_{y} \mid c_{y}
\end{array}\right)
$$

Table I shows our results testing the two algorithms on full resolution images with a special Region Of Interest (ROI) in the image. Both algorithms are not suitable for online data processing. The main drawback is that the processing time of the algorithm depends on the number of pixels. Therefore the number of pixels has to be reduced e.g. by selecting a small amount of interesting sections from the image. Instead of a random selection of Regions Of Interest (ROI) as proposed by Davision et al. [30], our approach is inspired by the biological process of searching for an object in a visual scene from humans [31, 32].

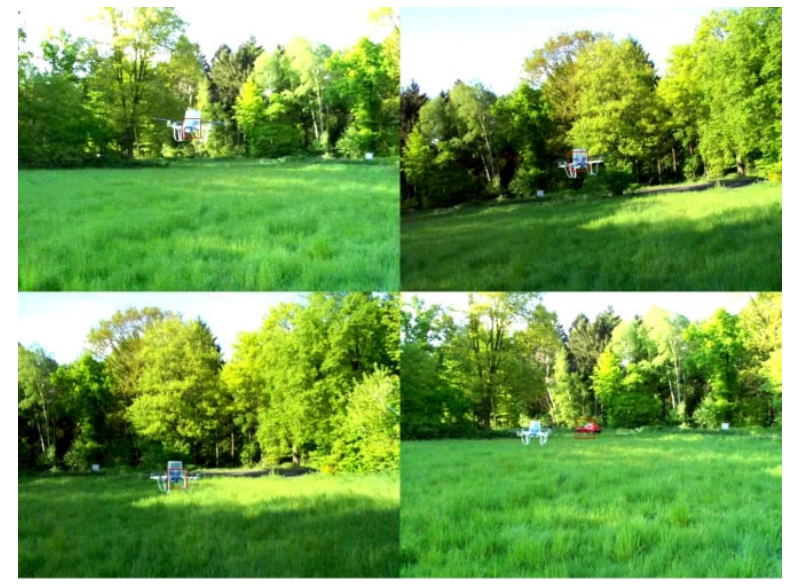

Figure 11. Most salient regions in four frames of the quadkopter flight. in more than $80 \%$ of the images is the quadkopter the most attentive object. When a red car drives through the scene the attention goes to it (bottom right).

\section{A. Human visual attention}

Human attention is caught by regions with objectspecific features such as color or orientations. The implemented visual attention system consists of a bottomup part computing data-driven saliency and a top-down part which enables goal-directed search [32]. The most salient regions are detected with respect to color, intensity and orientation. Bottom-up saliency results from uniqueness of features, e.g., a black sheep among white ones, whereas top-down saliency uses features that belong to a specified target, e.g., red when searching for a red ball. The bottom up part, is based on the well-known model of visual attention by Koch \& Ullman [33] used by many computational attention systems [34,35]. It computes saliencies according to the features intensity, orientation, and color and combines them in a saliency map. The most salient region in this map yields the focus of attention. The top-down part uses predefined feature weights to excite target-specific features and inhibit others e.g. for searching interesting red regions. On one hand the feature weights can be learned offline - as we presented in a previous paper with balls [36] - and on the other hand feature weights can be selected from a planning module to initiate a goal directed search. The important difference to our previous work is that we re-implemented the software and reduced the computation time from $10 \mathrm{~s}$ for a VGA picture to less than $50 \mathrm{~ms}$ [37]. That allows us to process all camera data online. Figure 8 shows four images of a teleoperated quadrotor flight marked with the bottom up saliency regions ${ }^{4}$. In the following we give a brief introduction to the visual attention system VOCUS

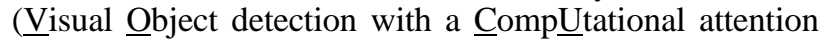
System) which detects these salient regions in images.

\section{1) Bottom-up saliency}

The first step for computing bottom-up saliency is to generate image pyramids for each feature to enable computations on different scales. Three features are considered: Intensity, orientation, and color. For the feature intensity, we convert the input image into grayscale and generate a Gaussian pyramid with 5 scales $\mathrm{s}_{0}$ to $\mathrm{s}_{4}$ by successively low-pass filtering and subsampling the input image, i.e., scale $(i+1)$ has half the width and height of scale $i$.

TABLE II.

GROUND TRUTH COMPARISON BETWEEN MEASURED DISTANCES AND CALCULATED DISTANCES WITH THE SURF ALGORITHM. IMAGES ARE TAKEN FROM PREDEFINED POSITIONS ALONG A LINE AND MATCHED TO THE IMAGE POSITION 0 .

\begin{tabular}{|c|c|c|}
\hline Position [cm] & $\begin{array}{c}\text { Number of matched } \\
\text { feature points }\end{array}$ & $\begin{array}{c}\text { Average } \\
\text { Deviation [\%] }\end{array}$ \\
\hline \hline 10 & 6 & 3.28 \\
\hline 20 & 6 & 1.92 \\
\hline 30 & 6 & 2.47 \\
\hline 50 & 5 & 1.09 \\
\hline 70 & 6 & 2.31 \\
\hline 100 & 4 & 1.89 \\
\hline 150 & 5 & 2.1 \\
\hline
\end{tabular}

The intensity maps are created by center-surround mechanisms, which compute the intensity differences between image regions and their surroundings. Two kinds of maps are computed, the on-center maps $I_{\text {on }}^{\prime \prime}$ for bright regions on dark background, and the off-center maps $I_{\text {off }}^{\prime \prime}$ : Each pixel in these maps is computed by the difference between a center $C$ and a surround $\sigma\left(I_{\text {on }}\right)$ or vice versa $\left(I_{\text {off }}^{\prime \prime}\right.$ ). Here, $C$ is a pixel in one of the scales $\mathrm{s}_{2}$ to $\mathrm{s}_{4}, \sigma$ is the average of the surrounding pixels for two different radii. This yields 12 intensity scale maps $I_{i, s, \sigma}^{\prime \prime}$ with $i \in\{$ on, off $\}, s \in\left\{s_{2}-s_{4}\right\}$, and $\sigma \in\{3,7\}$.

The maps for each $i$ are summed up by inter-scale addition $\oplus$, i.e., all maps are resized to scale 2 and then added up pixel by pixel yielding the intensity feature maps $I_{i}^{\prime}=\oplus_{s, \sigma} I_{i, s, \sigma}^{\prime \prime}$. To obtain the orientation maps, four oriented Gabor pyramids are created, detecting bar-like features of the orientations $\theta=\left\{0^{\circ}, 45^{\circ}, 90^{\circ}, 135^{\circ}\right\}$.

${ }^{4}$ A video with all images can be found under http://de.youtube.com/watch?v=qFS85rR1qGI. 
The maps 2 to 4 of each pyramid are summed up by interscale addition yielding 4 orientation feature maps $O_{\theta}^{\prime}$.

To compute the color feature maps, the color image is first converted into the uniform CIE LAB color space [38]. It represents colors similar to human perception. The three parameters in the model represent the luminance of the color (L), its position between red and green (A) and its position between yellow and blue (B). From the LAB image, a color image pyramid $P_{L A B}$ is generated, from which four color pyramids $P_{R}, P_{G}, P_{B}$ and $P_{Y}$ are computed for the colors red, green, blue, and yellow. The maps of these pyramids show to which degree a color is represented in an image, i.e., the maps in $P_{R}$ show the brightest values at red regions and the darkest values at green regions. Luminance is already considered in the intensity maps, so we ignore this channel here. The pixel value $P_{R, s}(x, y)$ in map s of pyramid $P_{R}$ is obtained by the distance between the corresponding pixel $P_{L A B}(x, y)$ and the prototype for red $r=\left(r_{a}, r_{b}\right)=(255,127)$. Since $P_{L A B}(x, y)$ is of the form $\left(p_{a}, p_{b}\right)$, this yields: $P_{L A B}(x, y)=\left\|\left(p_{a}, p_{b}\right)\right\|=\sqrt{\left(p_{a}-r_{a}\right)^{2}+\left(p_{b}-r_{b}\right)^{2}}$. On these pyramids, the color contrast is computed by oncenter-off-surround differences yielding 24 color scale maps $C_{\gamma, s, \sigma}^{\prime \prime}$ with $\gamma \in\{$ red, green, blue, yellow , $s \in\left\{s_{2}-s_{4}\right\}$, and $\sigma \in\{3-7\}$. The maps of each color are inter-scale added into 4 color feature maps $C_{\gamma}^{\prime}=\oplus_{s, \sigma} \mathcal{L}_{\gamma, s, \sigma}$.

\section{2) Fusing Saliencies}

All feature maps of one feature are combined into a conspicuity map yielding one map for each feature: $I=\sum_{i} W\left(I_{i}^{\prime}\right), \quad O=\sum_{\theta} W\left(O_{\theta}^{\prime}\right), \quad C=\sum_{\gamma} W\left(C_{\gamma}^{\prime}\right)$.

The bottom-up saliency map $S_{b u}$ is finally determined by fusing the conspicuity maps: $S_{b u}=W(I)+W(O)+W(C)$. The exclusivity weighting $W$ is a very important strategy since it enables the increase of the impact of relevant maps. Otherwise, a region peaking out in a single feature would be lost in the bulk of maps and no pop-out would be possible. In our context, important maps are those that have few highly salient peaks. For weighting maps according to the number of peaks, each map $M$ is divided by the square root of the number of local maxima $m$ that exceed a threshold $\quad t: W(M)=M / \sqrt{m} \quad \forall m: m>t$. Furthermore, the maps are normalized after summation relative to the largest value within the summed maps. This yields advantages over the normalization relative to a fixed value (details in [39]).

\section{3) The Focus of Attention (FOA)}

To determine the most salient location in $S_{b u}$, the point of maximal activation is located. Starting from this point, region growing recursively finds all neighbors with similar values within a threshold and the FOA is directed to this region. Finally, the salient region is inhibited in the saliency map by zeroing, enabling the computation of the next FOA.

\section{4) Search mode}

In search mode, firstly the bottom-up saliency map is computed. Additionally, we can determine a top-down saliency map that competes with the bottom-up map for saliency. The top-down map is composed of an excitation and an inhibition map. The excitation map $E$ is the weighted sum of all feature maps that are important for a goal directed search, namely the features with weights greater than 1 . The inhibition map $I$ contains the feature maps that are not present in the goal directed search, namely the features with weights smaller than 1 :

$$
\begin{gathered}
E=\frac{\sum_{i}\left(w_{i} \cdot \operatorname{Map}_{i}\right)}{\sum_{j}\left(w_{j}\right)} \forall i: w_{i}>1 \\
I=\frac{\sum_{i}\left(\left(1 / w_{i}\right) \cdot M a p_{i}\right)}{\sum_{j}\left(w_{j}\right)} \forall i: w_{i}<1
\end{gathered}
$$

The top-down saliency map $S_{(t d)}$ is obtained by: $S_{(t d)}=E-I$. The final saliency map $S$ is composed as a combination of bottom-up and top-down influences. When fusing the maps, it is possible to determine the degree to which each map contributes by weighting the maps with a top-down factor $t \in[0 . .1]: S=(1-t) \cdot S_{(b u)}+t \cdot S_{(t d)}$.

\section{B. First Results}

First results are shown in Table I. The well known feature extraction algorithms SIFT and SURF are not online capable in the current form. Furthermore, Table II indicates the precision of the feature extraction using the SURF algorithm. The robot is moved to several predefined positions and features are calculated in a specific ROI. Afterwards these features are matched to features in the initial image (at position 0). Based on the features the distances is calculated and compared to the real distance. The average error is around 3\%.

A second result is shown in figure 8 and the corresponding video which shows that VOCUS is an excellent appropriate for a selection of interesting regions, especially because of its real-time applicability (less then $40 \mathrm{~ms}$ for VGA images). The ROIs are used to guide the attention of the teleoperator in USAR missions and to track the quadrotor which is necessary for autonomous landing at the ground vehicle. Our experimental results show an accuracy of approx. 80\% while tracking the quadrotor. The omnidirectional view, the depth information in the images, the gained map and the ability of autonomous recovery of connection failures assists the operator in USAR scenarios.

\section{QUADROTOR}

This section focuses on the architecture of the Quadrotor. The hardware of the UAV was already 
described in section II. The UAV is an important component of the UAGV system which allows the operator to get a better overview of the environment. The problem which is addressed to the drones in USAR scenarios is a possibility for a single human to simultaneously manage the aerial vehicle and its camera [47]. This requires from the UAV to have some autonomous features to simplify the searching task of the personnel. This requirement and the necessity for the copter to accomplish tasks and return back or land safely in case of loosing a control signal is the basis for formulating the main goal of our project. The goal is to develop a multipurpose teleoperated VTOL platform with such autonomous features as automatic take-off and landing, position control, localization with return back function and obstacle avoidance. The platform should be suitable for both indoor and outdoor USAR applications and work in the collaboration with an UGV.

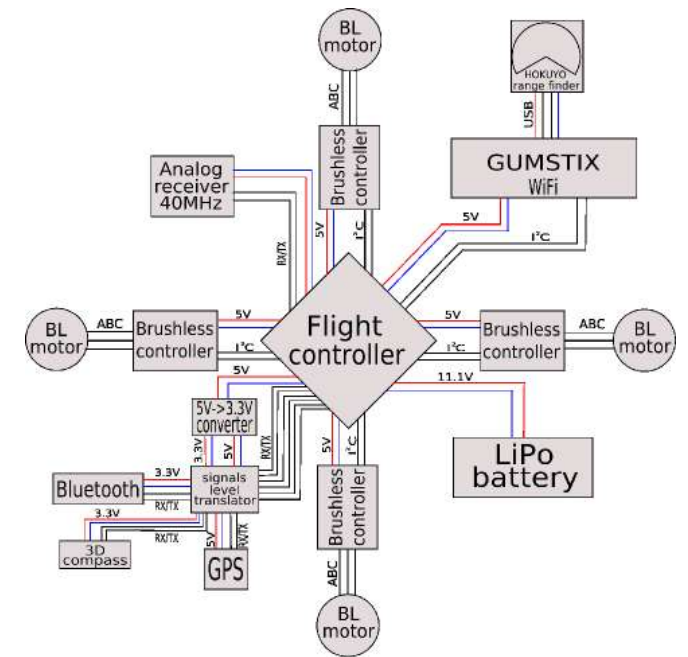

Figure 12. The quadrotors extended functional diagram. The flight control board utilizes the sensors data and computes the control signal for the brushless controllers. Communication between them and the gumstix computer is established via I2C bus. The signal level translator serves for normalizing different voltage levels of data signals.

\section{A. $\quad$ Related Work}

UAVs are classified in the two categories: Lighter-thenair vehicles and heavier-then-air crafts. Lighter-then-air UAVs (presented by mostly blimps) have two major disadvantages: size/payload proportion and low resistance to wind. However they also can be used in some Search an Rescue missions [48]. But they are not rapidly deployable and require carrying balloons with the gas along with the control equipment. Heavier-then-air vehicles are better in this case. Those which are used in USAR and WSAR (Wilderness Search and Rescue) are relatively small and can start without runways. Among them there are fixedwings airplanes, different modifications of helicopters and even kites [49]. Airplanes to compare with helicopters lack of maneuverability and mostly cannot hover (a hovering airplane is presented in [50]) but capable to carry higher payload on larger distances. These make them mostly applicable in WSAR scenarios or in exploration of wide open areas with very few obstacles. In USAR scenarios VTOL crafts with their high maneuverability ideally support rescue workers. They are able to start at very small open areas. Unmanned copters vary in size according to their application. Some are quite big and able to carry heavy high-resolution equipment [51] while others can be small.

Helicopters employed in USAR missions are also different in their design, starting from conventional ones and ending with quadrotors or octarotors (four or eight rotors helicopters). Quadrotors and their variations have advantages over conventional helicopters due to absence of complex mechanical control linkages for rotor actuation [52]. Instead they rely only on fixed pitch control. Furthermore, they are capable of changing the moving direction by varying only the motor's speeds. But that simplicity in mechanics leads to higher computational effort to stabilize this highly nonlinear model $[53,54]$.

Because of the mechanical simplicity and high maneuverability quadrotors became a field of interest of many researches. Especially this boom is seen for the last ten years when new brush-less motors and controllers became widely available on the market. These copters have enough pay load to carry the necessary equipment for surveillance and navigation and are relatively extendable to perform many tasks, including USAR scenarios.

The quadrotor has two pairs of counter-rotating, fixed pitch blades located at the four corners of the vehicle. Since the four-rotors helicopter is a highly non-linear and unstable platform and requires stability controllers to cope with its fast dynamics, the stabilization problem becomes a primary issue which is addressed in many articles [5358] and mostly overcame for some extend. Because of natural drift of the used stabilization sensors (gyroscopes and inertial sensors) and constant air moving (wind, convention currents) it is difficult to achieve a stable hovering for a long period of time.

This position drifting problem is manageable for outdoor environments by GPS [59-61]. However, in highly dense urban areas or for indoor-use GPS becomes inapplicable. There are also difficulties because of limited payload of UAVs and integrated hardware which does not allow the platform to be easily extended with additional sensors. Roberts et al. [62] uses a platform equipped with sonar for altitude control and four infrared range finders for hovering control. Matsue et al. [63] employed three infrared range sensors to measure the height above the ground and the distances to two perpendicular walls. The former platform showed good hovering results in empty rooms and was able to avoid large obstacles while the last one could follow walls. Kim [64] used 6 degrees of freedom inertial unit for conventional helicopter hovering stabilization.

Many approaches on the position drifting problem employ external sensing for position stabilization. Thus Castillo et. al [53] used Polhemus sensor for position tracking, Mori [65] processes on-board camera data on an external PC and Gurdan [66] performed experiments in a laboratory environment equipped with the indoor motion tracking system VICON that can measure the position vector of specific points on the body of the robot.

Although significant results were achieved, existing approaches lack of flexibility. Using external localization systems limits the copter's workspace to the area visible by that system. For avoiding collisions with obstacles and navigating in office buildings still no sufficient results have been shown. However for outdoor use the most 
promising solution is seen in employing GPS and 6-DOF Inertial Measurement Unit (IMU).

\section{B. UAV approach}

Selection of the proper platform for the research the choice fell to a non-commercial open-source project MikroKopter [6] with available preassembled flight and brushless control boards and open-source software. The flight control board contains a 3D accelerometer unit to calculate and align with the gravity of the earth. In order to provide automatic leveling of the copter, a complementary filter is implemented that processes the integrated angular velocity of three gyroscopes and the calculated Euler angles from the accelerometers. The output of the filter is used in a Proportional-IntegralDerivative (PID) controller.

To make the platform usable in USAR scenarios and to achieve a higher stability and functionality it is equipped with additional sensors and computational units. Figure 12 shows the functional diagram of the quadrotor used in the project. The main component is the flight controller in the middle of the diagram. The flight controller board with the pressure sensor for altitude control, gyros and an accelerometer is running on an Atmel ATMEGA644p micro-controller and communicates with the four brushless controllers via I2C bus. The brushless controllers in their turn are running on Atmel ATMEGA8 microcontroller and control the four brushless motors.

Rotation along central point (yaw) is eliminated with adding a 3D digital compass. Adding the compass is an important requirement for navigation. It allows us to fix the copter's orientation in space, in our case to north. Knowing the orientation we can use GPS coordinates for navigation and hovering stabilization. In our model we used standard u-blox GPS receiver reprogrammed for sending coordinates in autonomous mode with a $4 \mathrm{~Hz}$ frequency. GPS communication with the Flight Control board is established via second spare USART (Universal Synchronous/Asynchronous Receiver/Transmitter) of the AT-MEGA644p microcontroller.

The quadrotor is controlled via a $40 \mathrm{MHz}$ analog radio link. In order to achieve the vehicle's control compatibility with most electronic devices like laptops, mobile phones/smart-phones, pocket PCs etc. the quadrotor has been equipped with the Free2move Bluetooth module [67] and WiFi modules. The BlueTooth connection is performed via first USART of the on-board microcontroller and enables not only wireless data exchange but also program modification and flashing. Thus configuration parameters of the copter or the firmware can be changed "on-flight". The WiFi module is connected to a Gum-stix [68] embedded computer. Gumstix is a $600 \mathrm{MHz}$ microsize computer running with Linux OpenEmbedded operating system. In addition to three RS232 ports it has an USB host controller and slot for external memory (micro-SD). It doesn't require special power supply because it uses the $5 \mathrm{~V}$ which is standard for the helicopter board. The weight of the Gumstix computer doesn't exceed 100 grams. Gumstix communicates with the Flight Control board by mean of I2C bus connection.

The problems with possible loose of control signal and crashing to obstacles can be solved by extending the autonomy of the quadrotor. For this purpose a HOKUYO [69] laser range-finder is added. The laser scanner is needed for implementing collision avoidance function and will be used for indoor localization. Thus in case of loosing the control signal the helicopter should be able to find way back using built map and GPS position data. Data from the scanner is processed on the Gumstix computer. The collision avoidance function is served to prevent operator's error in USAR scenarios and focus him only on visual data from the on-board wireless camera. A comparison view on the HOKUYO range-finder, the wireless $2.4 \mathrm{GHz}$ analog camera and the Gumstix computer is presented on Fig. 13.

Since the Bluetooth device and the 3D compass need only $3 \mathrm{~V}$ power supply, a $5 \mathrm{~V}$ to $3.3 \mathrm{~V}$ voltage converter is implemented. Signals are then translated via a signal level translator to satisfy flight-control microprocessor's requirements.

\section{First results}

At the current state of the project we have achieved significant altitude and attitude stability of the platform with 3-axes gyroscopes and accelerometers and an air pressure sensor. The copter is remotely controlled and able to fly indoor and outdoor with just little adjustment of the flight trajectory by the operator. Altitude control is performed automatically according to the set point defined by the operator. Also by mean of the $3 \mathrm{D}$ electronic compass the orientation is automatically controlled and turns the copter to north after taking off.

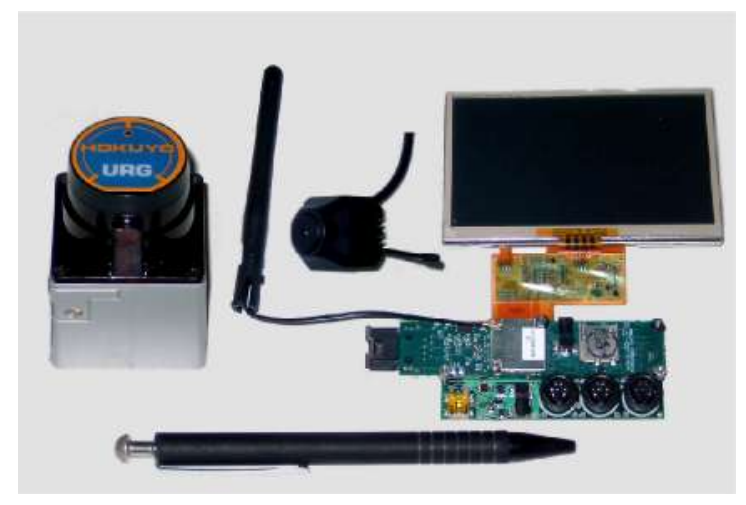

Figure 13. From the left to the right a HOKUYO URG-04lx laser rangefinder, a wireless visortech GP-811t camera and Gumstix Verdex XL6P computer with 802.11 (g) wireless communication module are shown. The display is detachable and is not needed during the flight.

Experiments in the robotic laboratory at Fraunhofer Campus Birlinghoven showed some drawbacks of using the magnetic compass. Multiple power cables under the floor induce unstable magnetic vectors in nearly all directions. It caused the copter to search for north orientation and confused the operator. Outdoor the performance shows excellent results. Manual landing on a small platform has also successfully tested at indoor and outdoor environments. Figure 14 shows the current prototype of the quadcopter ${ }^{5}$.

${ }^{5}$ Video links:
$\frac{\text { http://www.youtube.com/watch?v=48WVz9cEir8, }}{\text { http://de.youtube.com/watch?v=476uiL7ouO0, }}$
$\underline{\text { http://de.youtube.com/watch?v=Kh1SSfB2a5o }}$




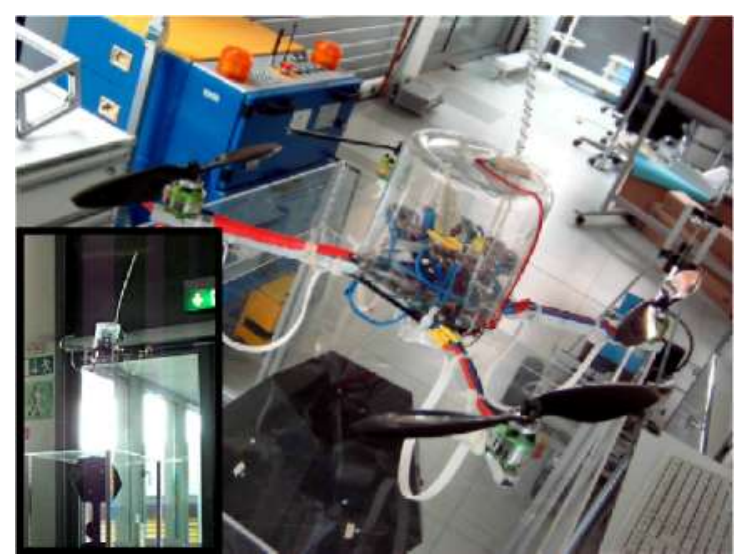

Figure 14. A prototype of the quadrotor used for the evaluation. On the bottom-left figure the copter is tested for landing on a small platform in the Fraunhofer IAIS robotic laboratory.

GPS tests showed good results on the field. The helicopter was able to cope with wind and hover at one point with error up to 0.5 meter with 6 to 8 satellites in the field of view of the GPS sensor. In the campus area with low altitude $(0.5 \mathrm{~m})$ the number of satellites captured by the copter varied from 2 to 5 . This leads to large 3D position errors up to 4 meters. The error is magnified by the fast dynamics of the quadrotor. The helicopter flying outdoor in the IAIS campus is shown in Fig. 15. From the picture we can see density of buildings in the testing area. With higher buildings the situation can be even worse.

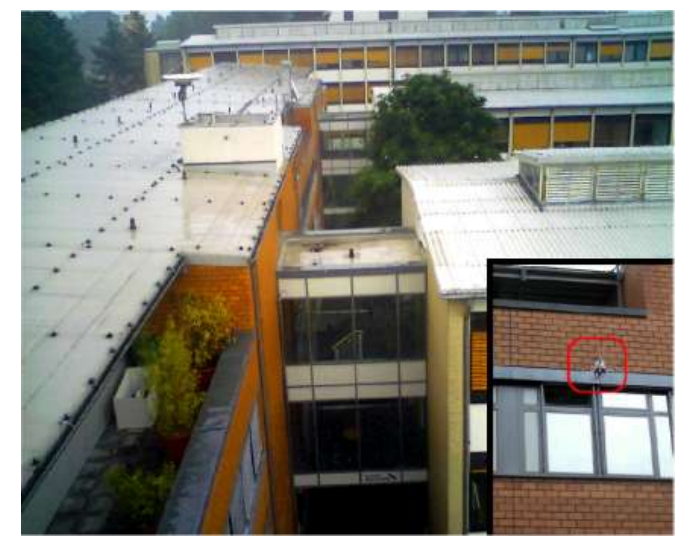

Figure 15. The quadrotor (red square) is flying above the roofs and near a window at Fraunhofer IAIS campus. The large picture is taken from the on-board camera. The figure illustrates possible search scenario in urban area like acquiring an interior overview through windows or scenery overview.

As shown by the experiments the position drifting problem cannot be totally eliminated with the chosen set of sensors. Since the platform can suffer from different deformations, external forces and control signal noise, precise calibration on horizontal position does not significantly reduce the drift. It is obvious that the copter lacks of sensing along horizontal axes. GPS provides the solution but only for opened areas or high altitude flights.

Although significant results have been achieved, the copter still requires operator presence in visible distance, especially indoor. The quadrotor is a valuable supplement in an USAR scenario especially if it cooperates with an UGV system. A simple interface should be provided to control it from any mobile device or other platforms like
UGVs. This is a cooperative work within the UGAV project group.

\section{CONCLUSIONS AND FUTURE WORK}

This paper presented our robotic system UGAV consisting of two semi-autonomous robot platforms, an UGV (VolksBot) and an UAV (Quadrotor). Furthermore, we described three main topics of combined UGV and UAV. (1) The teleoperated control with cell or smart phones with the new concept of automatic configuration of the smart phone based on a RKI-XML description of the vehicle control capabilities. (2) The camera and vision system with the focus to real time feature extraction e.g. for the tracking of the UAV and (3) the architecture and hardware of the UAV. Needless to say a lot of work remains to be done:

1) A future version, the OCU will be able to use Bluetooth or telephone numbers and supports the searching for the best communication channel.

2) With the vision system we will build maps online. Since the spatial information of features is known, they will be transformed into landmarks. These landmarks are inserted into a global map representing the environment. With the help of the map the robot is able to localize itself by comparing the current feature bearings with the stored landmarks. This will be done by triangulation and a linear algebra approach. The obtained map is useful if the remote connection to the robot is lost. In future work the robot should be able to backtrack its path autonomously until it reaches a position where the connection can be reestablished. In case of a complete breakdown of communication the return to the initial position is feasible.

3) The quadrotor will be equipped with a HOKUYO laser range-finder pursue the goal of multiple obstacles avoidance along with solving the hovering drift problem and flying indoor. With more and more increasing density of integrated circuits and increasing computational power it becomes possible to overcome computational expenses for micro aerial vehicles.

4) With 3D laser scanner we will build geometric maps and models with textures and semantic information based on the feature extraction.

\section{ACKNOWLEDGMENT}

The authors would like to thank our Fraunhofer colleagues for their support namely Reiner Frings, Jochen Winzer, Stefan May, Paul Ploger and Kai Pervolz. This work was partly supported by the DFG grant CH 74/9-1 (SPP 1125).

\section{REFERENCES}

[1] R.R. Murphy, "Marsupial and shape-shifting robots for urban search and rescue", Intelligent Systems and Their Applications, IEEE [see also IEEE Intelligent Systems], vol. 15, no. 2, pp. 1419, Mar/Apr 2000.

[2] G. S. Sukhatme, J. F. Montgomery, and R. T Vaughan, Robot Teams: From Diversity to Polymorphism, chapter Experiments with Cooperative Aerial-Ground robots, pp. 345-368, A.K. Peters, Ltd., 2002.

[3] Thomas Wisspeintner, Walter Nowak, and Ansgar Bredenfeld, RoboCup 2005: Robot Soccer World Cup IX, vol. 4020, chapter 
VolksBot - A Flexible Component-Based Mobile Robot System, pp. 716-723, Springer Berlin Heidelberg, 2006.

[4] Fraunhofer Institut Intelligent Analyse und Informationssysteme (IAIS), "Volksbot", http://www.volksbot.de/, 2008.

[5] Fraunhofer Institut Intelligent Analyse und Informationssysteme (IAIS), "Volksbot", http://www.3d-scanner.net/, 2008.

[6] Holger Buss and Ingo Busker, "Mikrokopter", http://www.mikrokopter.de/, May 2008.

[7] Oliver Wulf and Bernardo Wagner, "Fast 3d-scanning methods for laser measurement systems", in International Conference on Control Systems and Computer Science (CSCS14), 2003.

[8] Andreas Nuchter, Kai Lingemann, and Joachim Hertzberg und Hart-mut Surmann., "6d slam for 3d mapping outdoor environments", Journal of Field Robotics (JFR) Special Issue on Quantitative Performance Evaluation of Robotic and Intelligent Systems, vol. 24, pp. 699 - 722, 2007.

[9] Hartmut Surmann, Andreas Nuchter, K. Lingemann, and J. Hertzberg, "6d slam - preliminary report on closing the loop in six dimensions", in Proc. of the 5th IFAC Symposium on Intelligent Autonomous Vehicles, June 2004.

[10] S. Tachi, "Real-time remote robotics-toward networked telexistence", Computer Graphics and Applications, IEEE, vol. 18, no. 6, pp. 6-9, Nov/Dec 1998. (doi:10.1109/38.734972)

[11] R.R. Murphy, "Trial by fire [rescue robots]", Robotics \& Automation Magazine, IEEE, vol. 11, no. 3, pp. 50-61, Sept. 2004. (doi:10.1109/MRA.2004.1337826)

[12] T. B. Sheridan, "Teleoperation, telerobotics and telepresence: A progress report", ControlEng. Practice., vol. 3, no. 2, pp. 205-214, February 1995. (doi:10.1016/0967-0661(94)00078-U)

[13] J. Casper and R.R. Murphy, "Human-robot interactions during the robot-assisted urban search and rescue response at the world trade center", Systems, Man, and Cybernetics, Part B, IEEE Transactions on, vol. 33, no. 3, pp. 367-385, June 2003.

[14] P. Fiorini and R. Oboe, "Internet-based telerobotics: problems and approaches", Advanced Robotics, 1997. ICAR '97. Proceedings. 8th International Conference on, pp. 765-770, July 1997.

[15] F. Adachi, "Wireless past and future- evolving mobile communications systems", IEICE Trans Fundam Electron Commun Comput Sci (Inst Electron InfCommun Eng), vol. E84-A, pp. 5560, 2001.

[16] W Jansen and R. Ayers, "Guidelines on Cell Phone Forensics", NIST Special Publication, vol. 800, pp. 101, May 2007.

[17] P.A. Roche, M. Sun, and R.J. Sclabassi, "Using a cell phone for bioteleme-try", Proceedings of the IEEE 31st Annual Northeast Bioengineering Conference, pp. 65 - 66, April 2005.

[18] A. Sekmen, A.B. Koku, and S. Zein-Sabatto, "Human robot interaction via cellular phones", Systems, Man and Cybernetics, 2003. IEEE International Conference on, vol. 4, pp. 3937-3942, Oct. 2003, ISBN: 0-7803-7952-7.

[19] B.A. Myers, J. Nichols, J.O. Wobbrock, and R.C. Miller, "Taking handheld devices to the next level", Computer, vol. 37, pp. 36-43, December 2004. (doi:10.1109/MC.2004.258)

[20] Bradley J. Betts, Robert W Mah, Richard Papasin, Rommel Del Mundo, Dawn M. McIntosh, and Charles Jorgensen, "Improving situational awareness for first responders via mobile computing", Technical memorandum, NASA Ames Research Center, Moffett Field, CA 94035-1000, March 2005.

[21] Andres Industries, ", http://www.andres-industries.de/, May 2008.

[22] W Webb, "From "cellphone" to "remote control on life": how wireless communications will change the way we live over the next 20 years", 2002 IEEE Radio Frequency Integrated Circuits (RFIC) Symposium, pp. 7 -11, June 2002.

[23] "Android - an open handset alliance project : What is android", http://code.google.com/android/what-is-android.html, May 2008.

[24] [24] KimTopley, J2ME in a Nutshell, O'Reilly, March 2002, ISBN: 0-596-00253-X.

[25] "Android - an open handset alliance project: Location-based service apis", http://code.google.com/android/toolbox/apis/lbs.html, May 2008.

[26] Diego Lpez de Ipia, Juan Ignacio Vzquez, Daniel Garcia, Javier Fern-ndez, Ivn Garca, David Sinz, and Aitor Almeida, "A Middleware for the Deployment of Ambient Intelligent Spaces", Ambient Intelligence in Everyday Life, vol. 3864, pp. 239-255, 2006. (doi: $10.1007 / 11825890 \quad 12$ )
[27] "Android - an open handset alliance project: Android emulator", http://code.google.com/android/reference/emulator.html, March 2008.

[28] "Volksbot videos" http://www.youtube.com/user/Volksbot, May 2008.

[29] J. Craighead, B. Day, and R. Murphy, "Evaluation of canestas range sensor technology for urban search and rescue and robot navigation", http://www.crasar.org/, 2006.

[30] R. Murphy, J. Casper, J. Hyams, M. Micire, and B. Minten, "Mobility and sensing demands in usar", Industrial Electronics Society, 2000. IECON 2000. 26th Annual Confjerence of the IEEE, vol. 1, pp. 138-142 vol.1, 2000.

[31] Robin R. Murphy, "Rescue robotics for homeland security", Communications of the ACM, Special Issue on Emerging technologies for homeland security, vol. 47, pp. 66-68, March 2004.

[32] David G. Lowe, "Distinctive image features from scale-invariant key-points", in International Journal of Computer Vision, November 2004, vol. 60, pp. 91-110.

[33] Herbert Bay, Tinne Tuytelaars, and Luc Van Gool, "Surf: Speeded up robust features", in Computer Vision ECCV2006. 2006, vol. 3951/2006, pp. 404-417, Springer Berlin / Heidelberg.

[34] R. Sim, P. Elinas, M. Griffin, and J. J. Little, "Vision-based SLAM using the Rao-Blackwellised particle filter", in Proceedings of the IJCAI Workshop on Reasoning with Uncertainty in Robotics (RUR), Edinburgh, Scotland, 2005, pp. 9-16.

[35] N Karlsson, "The vslam algorithm for robust localization and mapping", in Int. Conf. on Robotics and Automation, 2005.

[36] Hauke Strasdat, Cyrill Stachniss, Maren Bennewitz, and Wolfram Bur-gard, "Visual bearing-only simultaneous localization and mapping with improved feature matching", in Fachgesprche Autonome Mobile Systeme (AMS), 2007.

[37] Andrew J. Davison, Ian D. Reid, Nicholas D. Molton, and Olivier Stasse, "Monoslam: Real-time single camera slam", in TRANSAC TIONS ON PATTERN ANALYSI S AND MACHINE INTELLIGENCE, June 2007, vol. 29.

[38] U. Neisser, Cognitive Psychology, Appleton-Century-Crofts, N.Y., 1967.

[39] Simone Frintrop, VOCUS: A Visual Attention System for Object Detection and Goal-Directed Search, PhD thesis, University of Bonn, July 2005.

[40] C. Koch and S. Ullman, "Shifts in selective visual attention: towards the underlying neural circuitry", Human Neurobiology, pp. 219-227, 1985.

[41] L. Itti, C. Koch, and E. Niebur, "A model of saliency-based visual attention for rapid scene analysis", IEEE Trans. on Pattern Analysis \& Machine Intelligence, vol. 20, no. 11, pp. 1254-1259, 1998. (doi:10.1109/34.730558)

[42] G. Backer, B. Mertsching, and M. Bollmann, "Data- and modeldriven gaze control for an active-vision system", IEEE Trans. on Pattern Analysis \& Machine Intelligence, vol. 23(12), pp. 14151429, 2001. (doi:10.1109/34.977565)

[43] Sara Mitri, Simone Frintrop, Kai Pervolz, Hartmut Surmann, and Andreas Nuchter, "Robust Object Detection at Regions of Interest with an Application in Ball Recognition", in Proceedings IEEE 2005 International Conference Robotics and Automation (ICRA '05), Barcelona, Spain, April 2005, pp. 126-131, Conf-A.

[44] Stefan May, Maria Klodt, Erich Rome, and Ralph Breithaupt, "Gpu-accelerated affordance cueing based on visual attention", in Proceedings of the IEEE/RSJ International Conference on Intelligent Robots and Systems (IROS), San Diego, USA, 2007.

[45] R. E. Burger, Colormanagement. Konzepte, Begriffe Systeme, Springer, 1997.

[46] S. Frintrop, E. Rome, A. Nuchter, and H. Surmann, "A bimodal laser-based attention system", J. of Computer Vision and Image Understanding (CVIU), Special Issue on Attention and Performance, 2005.

[47] J. Cooper and M.A. Goodrich, "Towards combining UAV and sensor operator roles in UAV-enabled visual search", Proceedings of the 3rd international conference on Human robot interaction, pp. 351-358, 2008.

[48] KW Sevcik, WE Green, and PY Oh, "Exploring search-and-rescue in near-earth environments for aerial robots", Advanced Intelligent Mecha-tronics. Proceedings, 2005 IEEE/ASME International Conference on, pp. 699-704, 2005.

[49] W.E. Green and P.Y. Oh, "An Aerial Vision Platform for Acquiring Sit-uational Awareness", Proc. Int. Conf. Computer, 
Communication, and Control Technologies, vol. 5, pp. 289-295, 2003.

[50] W. E. Green and P. Y. Oh, "A fixed-wing aircraft for hovering in caves, tunnels, and buildings", American Control Conference, 2006, p. 6, 2006.

[51] P. Doherty and P. Rudol, "A UAV Search and Rescue Scenario with Human Body Detection and Geolocalization", LECTURE NOTES IN COMPUTER SCIENCE, vol. 4830, pp. 1, 2007. (doi:10.1007/978-3-540-76928-6_1)

[52] G.M. Hoffmann, H. Huang, S.L. Waslander, and C.J. Tomlin, "Quadrotor Helicopter Flight Dynamics and Control: Theory and Experiment", Proc. AIAA Guidance, Navigation, and Control Conf., Hilton Head, SC, August, 2007.

[53] P. Castillo, A. Dzul, and R. Lozano, "Real-time stabilization and tracking of a four-rotor mini rotorcraft", Control Systems Technology, IEEE Transactions on, vol. 12, no. 4, pp. 510-516, 2004. (doi:10.1109/TCST.2004.825052)

[54] A. Mokhtari and A. Benallegue, "Dynamic feedback controller of Euler angles and wind parameters estimation for a quadrotor unmanned aerial vehicle", Robotics and Automation, 2004. Proceedings. ICRA'04. 2004 IEEE International Conference on, vol. 3, pp. 2359-2366, 2004.

[55] P. Pounds, R. Mahony, and P. Corke, "Modelling and Control of a Quad-Rotor Robot", Proceedings of the Australasian Conference on Robotics and Automation, 2006.

[56] P. McKerrow, "Modelling the Draganflyer four-rotor helicopter", Robotics and Automation, 2004. Proceedings. ICRA'04. 2004 IEEE International Conference on, vol. 4, pp. 3596-3601, 2004.

[57] L. Beji and A. Abichou, "Streamlined Rotors Mini Rotorcraft: Trajectory Generation and Tracking", International Journal of Control, Automation, and Systems, vol. 3, no. 1, pp. 87-99, 2005.

[58] S. Bouabdallah, P. Murrieri, and R. Siegwart, "Design and control of an indoor micro quadrotor", Robotics and Automation, 2004. Proceedings. ICRA'04. 2004 IEEE International Conference on, vol. 5, pp. 4393-4398, 2004.

[59] "Microdrones gmbh", http://www.microdrones.com, May 2008.

[60] O. Meister, R. Monikes, J. Wendel, N. Frietsch, C. Schlaile, and G.F. Trommer, "Development of a GPS/INS/MAG navigation system and way-point navigator for a VTOL UAV", Proceedings ofSPIE, vol. 6561, pp. 6561 1D, 2007.

[61] E. Courses and T. Surveys, "Enhancement of GPS Signals for Automatic Control of a UAV Helicopter System", Control and Automation, 2007. ICCA 2007. IEEE International Conference on, pp. 1185-1189, 2007.

[62] J.F. Roberts, T. Stirling, J.C. Zufferey, and D. Floreano, "Quadrotor Using Minimal Sensing For Autonomous Indoor Flight", European Air Vehicle Conference and Flight Competition EMAV2007, 2007.

[63] A. Matsue, W. Hirosue, H. Tokutake, S. Sunada, and A. Ohkura, "Navigation of Small and Lightweight Helicopter", Trans. Jpn. Soc. Aeronaut. Space Sci, vol. 48, pp. 177-479, 2005. (doi:10.2322/tjsass.48.177)

[64] B. KIM, Y CHANG, and M.H. LEE, "System Identification and 6DOF Hovering Controller Design of Unmanned Model Helicopter", JSME International Journal Series C, vol. 49, no. 4, pp. 1048-1057, 2006. (doi:10.1299/jsmec.49.1048)

[65] R. Mori, T. Kubo, and T. Kinoshita, "Vision-Based Hovering Control of a Small-Scale Unmanned Helicopter", SICE-ICASE, 2006. International Joint Conference, pp. 1274-1278, 2006.

[66] D. Gurdan, J. Stumpf, M. Achtelik, K.M. Doth, G. Hirzinger, and D. Rus, "Energy-efficient Autonomous Four-rotor Flying Robot Controlled at $1 \mathrm{kHz}$ ", Robotics and Automation, 2007 IEEE International Conference on, pp. 361-366, 2007.

[67] "Technical description of free2move bluetooth module f2m03gx / gxa", http://www.free2move.se/pdf/F2M03GXGXA.pdf, May 2008.

[68] "gumstix inc.", http://www.gumstix.com/, May 2008.

[69] "Hokuyo automatic co. ltd.",http://www.hokuyoaut.jp/02sensor/07scanner/urg.html, May 2008.

\section{AUTHORS}

Hartmut Surmann is a Senior Researcher and Scientist at the Fraunhofer Institut fürIntelligente Analyseund Informationssysteme (IAIS). His primary research interests are autonomous mobile robotics and computational intelligence. He received several awards, e.g.,The FUZZ-IEEE/IFES'95 robot intelligence award, NC2001 best presentation award, SSRR 2005 best paper award and the Ph.D. award for his thesis from the German AI institutes in 1996. His robot KURT3D won the second place in the RoboCup rescue robot league at the world championship in Lisbon in 2004 (e-mail: hartmut.surmann@iais.fraunhofer.de,web: http://www.iai s.fraunhofer.de/surmann.html).

Dirk Holz is with the Fraunhofer Institute for Intelligent Analysis and Information Systems (IAIS) and the Computer Science Department of the Bonn-RheinSieg University of Applied Sciences, Sankt Au-gustin, Germany. He received his Diploma in Computer Science from the University of Applied Sciences Cologne and is currently working on his Master Thesis in Autonomous Systems (e-mail: dirk.holz@inf.fh-brs.de).

Sebastian Blumenthal is a master student of Autonomous Systems at the Department of Computer Science, University of Applied Sciences Bonn-RheinSieg, Sankt Augustin, Germany. He received his Bachelor of Science in Computer Science from the University of Applied Science Bonn-Rhein-Sieg, Germany in 2007. He is currently working in a research and developement project on visual odometry in cooperation with the Fraunhofer IAIS. (web: http://www2.inf.fhbrs.de/ sblume2s, mail: sebastian.blumenthal.2004W@informatik.fh-bonn-rheinsieg.de,)

Thorsten Linder is a master student of Autonomous Systems at the Department of Computer Science, University of Applied Sciences Bonn-Rhein-Sieg, Sankt Augustin, Germany. He received his Bachelor of Science in Computer Science from the University of Applied Science Bonn-Rhein-Sieg, Germany in 2007. He is currently working in a research and developement project on teleoperation with mobile devices in cooperation with the Fraunhofer IAIS (e-mail: thorsten.linder.2004W@informatik.fh-bonn-rhein-sieg.de, web: http://www2.inf. fh-brs.de/ tlinde2s).

Peter Molitor is a student at the Cologne University of Applied Sciences in the diploma course "Technical Informatics". He is currently working on his diploma thesis on visual robot-navigation in cooperation with the Fraunhofer IAIS. His primary research interests are microcontrollers, embedded systems, autonomous systems and vision systems, (e-mail: research @molitor.biz, web: http://molitor.biz).

Viatcheslav Tretyakov is a master student of Autonomous Systems at the Department of Computer Science, University of Applied Sciences Bonn-RheinSieg, Sankt Augustin, Germany. He received his Diploma (Engineer) in Industrial Automation from Magnitogorsk State Technical University, Russia in 2004. He is currently working in a research and developement project on autonomous unmanned aerial vehicles in cooperation with the Fraunhofer IAIS (e-mail: viatcheslav.tretyakov.2007W @ informatik.fh-bonn-rheinsieg.de).

Manuscript received 21 October 2008. Published as submitted by the authors. 